\title{
Antipsychotic Medication in Schizophrenic Patients is Associated with Higher Risks of Developing Bone Fractures and Refractures
}

\author{
Ching-Min Kuo ${ }^{1, *}$, Wei-Jen Liao ${ }^{2, *}$, Chun-Che Huang ${ }^{3,4}$, Tsuo-Hung Lan ${ }^{1,5}$, Ching-Heng Lin $^{4}$, \\ Shun-Ping Wang ${ }^{2,6}$, Cheng-Hung Lee ${ }^{2}$, Ping-Wing Lui ${ }^{7,8}$
}

Departments of ${ }^{1}$ Psychiatry and ${ }^{2}$ Orthopedics, Taichung Veterans General Hospital, Taichung, ${ }^{3}$ Department of Healthcare Administration, I-Shou University, Kaohsiung, ${ }^{4}$ Health Medical Research, Taichung Veterans General Hospital, Taichung, ${ }^{5}$ Tsaotun Psychiatric Center, Ministry of Health and Welfare, Nantou, ${ }^{6}$ Sports Recreation and Management Continuing Studies-Bachelor's Degree Completion Program, Tunghai University, ${ }^{7}$ Department of Anesthesiology, Taichung Veterans General Hospital, Taichung, ${ }^{8}$ Office of Superintendent, Saint Paul Hospital, Taoyuan, Taiwan

Objective: The relationship of antipsychotics and the risk of refracture in treated patients is unclear. The aim of this study is to evaluate the association between prolonged antipsychotic and the incidences of bone fractures and refractures in schizophrenia.

Methods: This is a retrospective nested case-control study using Taiwan National Health Insurance Research Database recorded from 2000 to 2005, with cases followed up to end of 2011. Total of 7,842 schizophrenic patients, 3,955 had developed bone fractures were compared with 3,887 control subjects matched in age, sex, and index date. Antipsychotic drug exposure was classified based on the drug type and medication duration. Conditional logistic regression analyses were performed. Odds ratio (OR) and confidence interval $(\mathrm{Cl})$ were calculated.

Results: We found (after adjustments) higher risks of developing fractures under continued use of typical $(\mathrm{OR}=1.70$; $95 \% \mathrm{Cl}, 1.51-1.91)$ or atypical antipsychotics $(\mathrm{OR}=1.43 ; 95 \% \mathrm{Cl}, 1.28-1.60)$ were found. Additionally, continued use typical $(\mathrm{OR}=1.84 ; 95 \% \mathrm{Cl}, 1.35-2.50)$ or atypical antipsychotics $(\mathrm{OR}=1.44 ; 95 \% \mathrm{Cl}, 1.06-1.95)$ was positively associated with refracture risks. Moreover, refractures were associated with continuous use of chlorpromazine (one typical antipsychotics, $\mathrm{OR}=2.45 ; 95 \% \mathrm{Cl}, 1.14-5.25)$, and risperidone $(\mathrm{OR}=1.48 ; 95 \% \mathrm{Cl}, 1.01-2.16)$ or zotepine $(\mathrm{OR}=2.15 ; 95 \% \mathrm{Cl}, 1.06-4.36)$ (two atypical antipsychotics).

Conclusion: Higher risks of bone fracture and refracture were found in schizophrenia under prolonged medication with typical or atypical antipsychotics. We therefore recommend that clinicians should pay more attention on bone density monitoring for patients using long-term antipsychotics.

KEY WORDS: Antipsychotic agents; Fracture; Refracture; Schizophrenia.

\section{INTRODUCTION}

Schizophrenia is a major neuropsychiatric disorder posing a considerable societal burden. It has estimated a lifetime prevalence from 0.30 to $0.66 \%$ per 100,000 population [1], and mean life prevalence is just below $<1 \%$ worldwide [2] and $0.44 \%$ in Taiwan [3]. Regional differ-

Received: February 19, 2020 / Revised: April 20, 2020

Accepted: May 27, 2020

Address for correspondence: Ping-Wing Lui

Department of Anesthesiology, Taichung Veterans General Hospital, No. 1650, Sec. 4, Taiwan Blvd, Xitun Dist, Taichung, Taiwan

E-mail: superanesthesia@gmail.com

ORCID: https://orcid.org/0000-0002-2918-2413

*These authors contributed equally to this study. ences of prevalence exist across different cities and varied according to the degree of urbanization $[2,3]$. It has been well documented that antipsychotic drugs are commonly used in clinical practice as major treatment in schizophrenia.

Several factors related to antipsychotic use and fracture risks have been reported [4]. These factors including falls, vitamin $\mathrm{D}$ deficiency, low bone mineral density (BMD), and low body mass index. With short-term uses of antipsychotics (including those for sedation), higher risks of fracture are potentially caused by extrapyramidal symptoms, and orthostatic hypotension [5]. In addition, long-term antipsychotic use elevates serum levels of prolactin [6], which accelerates bone loss and in turn reduces BMD, another risk factor for bone fracture [7]. Bone fractures

(c) This is an Open-Access article distributed under the terms of the Creative Commons Attribution Non-Commercial License (http://creativecommons.org/licenses/by-nc/4.0) which permits unrestricted non-commercial use, distribution, and reproduction in any medium, provided the original work is properly cited. 
could increase morbidity and mortality, or cause long-term dependency. Fracture is also an important public health issue as it lowers the life quality and increases health care costs.

A recent meta-analysis on 34 studies by Papola et al. [8], reported that antipsychotic use is associated with higher risks of hip fracture, as well as all fractures. However only few studies have evaluated in the elderly, antipsychotic use and the development of hip fracture $[9,10]$. The influence of continuous antipsychotic therapy on refracture remains unclear. Therefore, this study aimed to investigate the association between continuous antipsychotic treatments and risks of fracture and refracture among schizophrenic patients.

\section{METHODS}

\section{Data Source}

In this retrospective population-based study, we used the Registry for Catastrophic Illness Patient Database (RCIPD), a part of the Taiwan's National Health Insurance Research Database (NHIRD). The NHI program that was launched in March 1995 covers 99\% of the 23 million Taiwanese population. The schizophrenia cases in the RCIPD were validated by certified psychiatrists during regulatory reviews and verification of medical records rigorously conducted by the National Health Insurance Administration (NHIA). All patient identification numbers were encrypted. The database included information on demographics, outpatient, inpatient, and drug prescription claims for beneficiaries with catastrophic illnesses. Clinical diagnoses were classified according to the International Classification of Diseases, 9th Revision, Clinical Modification (ICD-9-CM) coding. Our study was approved by the Institutional Review Board of Taichung Veterans General Hospital (approval no. CE13151B-5).

\section{Study Design}

The nested case-control study was conducted over a 10-year period from 2000 to 2011. Patient included in the analysis were registered between 2000 and 2005, and our observation period was 6-years longer (from 2000 to 2011). The exclusion criteria were: $<18$ or $>120$ years-old; or those who had preceding fractures (ICD-9-CM 800829) prior to year of 2000. This ensured the study was conducted on adults with new-onset fractures. In addi- tion, patients who did not receive antipsychotic medications were also excluded.

\section{Cases and Control Definitions for Fractures}

Patients with schizophrenia (ICD-9-CM 295) who were newly diagnosed with a fracture during the observation period formed the case group. A fracture event was based on the ICD-9-CM diagnostic codes (800-829) with at least one hospitalization, or outpatient diagnosis by an orthopedist to ensure accurate diagnosis. The index date was the date when the first fracture was diagnosed, it may diagnosed via physical examination, $\mathrm{X}$-ray, computed tomographic or even magnetic resonance imaging image studies. A fracture event preceded the date of first antipsychotic prescription.

The control group was composed of patients with no fracture diagnosis during the observation period formed the control group. Control patients were excluded if they died before the index date, or whose index dates were before the diagnosis of schizophrenia (or mood disorder). For each case with fracture, we randomly selected one control without fracture and conducted a 1:1 frequency match based on sex, age (year), and index date. The flow diagram of sample selection is showed in Figure 1.

\section{Cases and Control Definitions for Refractures}

Among those with a fracture, patients who had fracture diagnostic codes at least 180 days after the initiation of fracture were classified as refracture episodes (cases). In addition, the date of first refracture was defined as the index date. Controls were patients who did not have refracture during the observation period. Control patients who died before the index date, or whose index date was before the date of initial fracture, were excluded. The control was randomly selected from patients without refractures using a 1:1 ratio of frequency match based on sex, age, and index date. The flow diagram of sample selection for the refracture is showed in Figure 2.

\section{Antipsychotic Medication Exposures}

Information regarding antipsychotic use was obtained from the prescription records. We evaluated effects of antipsychotics according to their patterns of medication use (i.e., continue users vs. past users). The temporal windows of exposure in terms of individual antipsychotic medications were classified into two groups: continue and past 


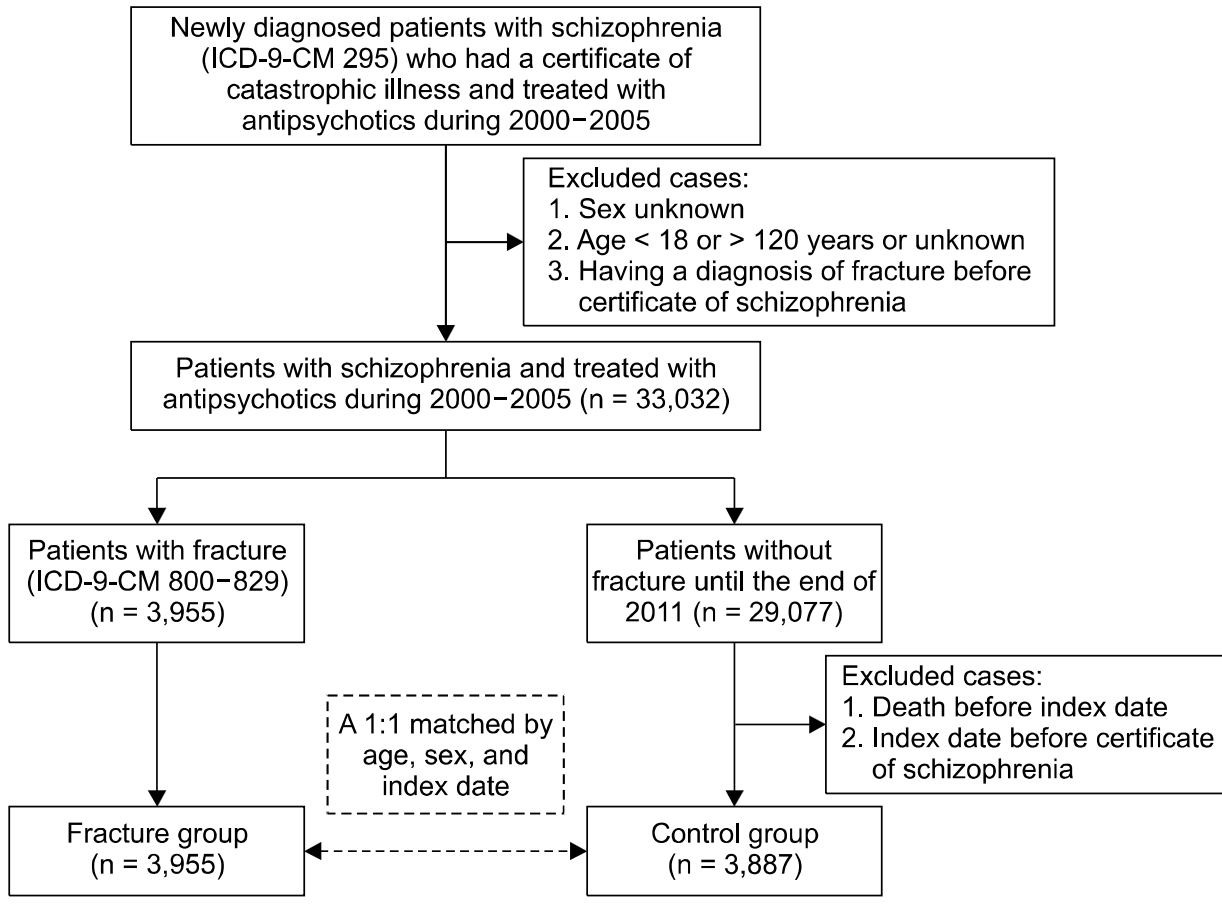

Fig. 1. Flow chart of sample selection for fracture patients. ICD-9-CM, International Classification of Diseases, 9th Revision, Clinical Modification.

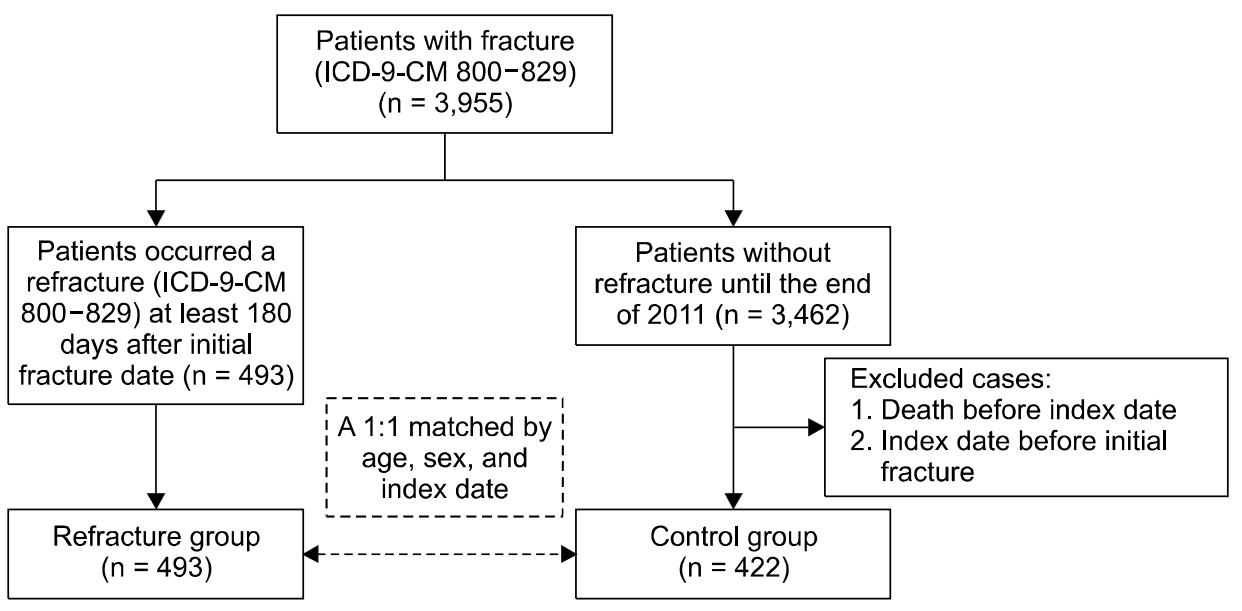

Fig. 2. Flow chart of sample selection for refracture patients. ICD-9-CM, International Classification of Diseases, 9th Revision, Clinical Modification. users. Continue user was defined as patients receiving antipsychotic prescription both within 90 days and $>90$ days before the index date. Past user was defined as patients receiving antipsychotic $>90$ days before index date, but not within 90 days. In order to estimate the effect of current psychopharmacological treatment, risk of fracture increased from the first days of use antipsychotic and increase thereafter, medication status was based on prescriptions picked up from the pharmacy within the last 3 months before index date [10-12]. Based on our study design, the data extracted from Taiwan National Health Insurance Research Database were analyzed. The first-line recommended medication for schizophrenia is antipsychotic, and Taiwanese National Health Insurance (NHI) cover the prescription, so few patients do not use the prescribed antipsychotic.

Antipsychotic medications were grouped based on the classification scheme of the Anatomic Therapeutic Chemical (ATC), as developed by the World Health Organization Collaborating Center. Based on the mechanisms of action, medications were further divided into typical and atypical antipsychotics [12]. Typical antipsychotics included the following: chlorpromazine (ATC code N05AA01), levomepromazine (N05AA02), prochlorperazine (N05AB04), 
trifluoperazine (N05AB06), perphenazine (N05AB03), fluphenazine (N05AB02), thioridazine (N05AC02), pipotiazine (N05AC04), chlorprothixene (N05AF03), thiothixene, clopenthixol (N05AF02), flupentixol (N05AF01), zuclopenthixol (N05AF05), droperidol (N05AD08), haloperidol (N05AD01), penfluridol (N05AG03), moperone (N05AD04), pimozide (N05AG02), loxapine (N05AH01), sulpiride (N05AL01), and clothiapine (N05AH06). Atypical antipsychotics included clozapine (N05AH02), risperidone (N05AX08), paliperidone (N05AX12), ziprasidone (N05AE04), olanzapine (N05AH03), quetiapine (N05AH04), amisulpiride (N05AL05), zotepine (N05AX11), and aripiprazole (N05AX12).

\section{Covariates}

The covariate variables included patient's age, sex, and comorbid conditions. The medical comorbidity was based on the Charlson comorbidity index $(\mathrm{CCl})$, this has been widely used in previous studies $[12,13]$. The comorbid conditions were the following: diabetes mellitus, hypertension, congestive heart failure, chronic obstructive pulmonary disease, chronic kidney disease, liver cirrhosis, autoimmune disease, cerebrovascular accident, and ischemic heart disease [13].

\section{Statistical Analyses}

Distributions of demographics, $\mathrm{CCl}$, and antipsychotic medication use between the case and control groups were examined using the Student $t$ test for continuous variables, and chi-square $\left(\chi^{2}\right)$ or Fisher's exact test for categorical variables. In addition, conditional univariate and multivariate logistic regression models were used to estimate the effect of typical and atypical antipsychotics on the risks of fracture and refracture based on the medication use (continue vs. past use).

Subgroup analyses were performed to estimate effects of fracture and refracture developments according to different typical and atypical antipsychotics. Multivariate conditional logistic regression models were used. Odds ratio (OR) and 95\% confidence intervals $(\mathrm{Cl})$ were determined. Statistical significance was set at $p<0.05$. All analyses were performed using SAS 9.4 version for Windows (SAS Institue, Cary, NC, USA).

This is a retrospective nested case-control study using the Taiwan National Health Insurance Research Database recorded from 2000 to 2005, with the cases followed up to the end of 2011. The risk of 3,955 schizophrenia with antipsychotic use had developed bone fractures at first step matched in age, sex, and index date were compared. Then the antipsychotic medications and risks of refracture were analyzed in the secondary steps. Antipsychotic drug exposure was classified based on the drug type and the medication duration. Conditional logistic regression analyses were performed. $\mathrm{OR}$ and $\mathrm{Cl}$ were calculated.

\section{RESULTS}

Antipsychotic Use and Risks of Fracture Development A total of 7,842 patients with schizophrenia $(3,955$ in the fracture group and 3,887 in the control group) were analyzed. Table 1 shows the distributions of demographic characteristics, $\mathrm{CCl}$, and types of antipsychotic prescribed for the two groups. The mean age of the case (fracture) group was $41.7 \pm 15$ years, and of the control group, $41.4 \pm$ 14.6 years. Patients in the fracture group had more physical comorbidities based on $\mathrm{CCl}(p<0.001)$, may represented more co morbidity. In addition, continuous use of antipsychotics was found in $78 \%$ of fracture patients, and $68.8 \%$ of controls $(p<0.001)$. Stratified analyses showed for the fracture group, $46.8 \%$ of the patients had a continuous use of typical antipsychotics, and $62.2 \%$ had atypical antipsychotics. For controls, these respective numbers were $38.1 \%$ and $55.1 \%$ (both $p<0.001$ ) (Table 1). Although the atypical antipsychotic were prescribed more for Schizophrenia patients in both groups, but the result showed the positive correlation between antipsychotic use and higher fracture risk.

Table 2 summarizes the relationship we found between fracture development and antipsychotic types. In comparison with past users, the risk of fracture development was higher in continuous users, after adjusting for other confounding factors $(\mathrm{OR}=1.55 ; 95 \% \mathrm{Cl}, 1.40-1.72)$. We similarly found higher odds for developing fractures in continuous use of antipsychotics, regardless of typical $(\mathrm{OR}=1.70 ; 95 \% \mathrm{Cl}, 1.51-1.91)$ or atypical $(\mathrm{OR}=1.43$; $95 \% \mathrm{Cl}, 1.28-1.60)$ (Table 2).

\section{Prior Fracture and Antipsychotic Use in Association with Risks of Refracture}

We analyzed a total of 915 schizophrenic patients who had previous fractures: 493 in the refracture group and 422 in the control group. Their mean age was $41.7 \pm 15.0$ 
Table 1. Characteristics of schizophrenia patients with and without fractures

\begin{tabular}{|c|c|c|c|c|c|}
\hline Variable & $\begin{array}{l}\text { Without fracture } \\
\qquad(\mathrm{n}=3,887)\end{array}$ & $\begin{array}{l}\text { With fracture } \\
(\mathrm{n}=3,955)\end{array}$ & $p$ value & $\begin{array}{c}\text { Crude OR } \\
(95 \% \mathrm{Cl})\end{array}$ & $p$ value \\
\hline Age (yr) & $41.4 \pm 14.6$ & $41.7 \pm 15.0$ & $0.363^{*}$ & $1.00(1.00-1.01)$ & 0.363 \\
\hline Sex & & & 0.646 & & \\
\hline Female & $1,747(44.9)$ & $1,798(45.5)$ & & 1.00 & \\
\hline Male & $2,140(55.1)$ & $2,157(54.5)$ & & $0.98(0.90-1.07)$ & 0.646 \\
\hline $\mathrm{CCl}$ & & & $<0.001$ & & \\
\hline 0 & $2,868(73.8)$ & $2,525(63.8)$ & & 1.00 & \\
\hline $1-2$ & $859(22.1)$ & 1,151 (29.1) & & $1.52(1.37-1.69)$ & $<0.001$ \\
\hline$\geq 3$ & $160(4.1)$ & $279(7.1)$ & & $1.98(1.62-2.42)$ & $<0.001$ \\
\hline \multicolumn{6}{|l|}{ Use of antipsychotics } \\
\hline Typical and/or atypical & & & $<0.001$ & & \\
\hline Past use & $1,202(31.2)$ & $860(22.0)$ & & 1.00 & \\
\hline Continue use & 2,655 (68.8) & $3,054(78.0)$ & & $1.61(1.45-1.78)$ & $<0.001$ \\
\hline Typical & & & $<0.001$ & & \\
\hline Past use & $2,320(61.9)$ & $2,041(53.2)$ & & 1.00 & \\
\hline Continue use & $1,427(38.1)$ & $1,792(46.8)$ & & $1.43(1.30-1.56)$ & $<0.001$ \\
\hline Atypical & & & $<0.001$ & & \\
\hline Past use & $1,251(44.9)$ & 1,105 (37.8) & & 1.00 & \\
\hline Continue use & $1,538(55.1)$ & $1,819(62.2)$ & & $1.34(1.20-1.49)$ & $<0.001$ \\
\hline
\end{tabular}

Values are presented as mean \pm standard deviation or number (\%).

$\mathrm{OR}$, odds ratio; $\mathrm{Cl}$, confidence interval; $\mathrm{CCl}$, Charlson comorbidity index.

${ }^{*} t$ test; chi-squared test or Fisher's exact test for all other $p$ value.

Table 2. Adjusted odds ratio (OR) of fractures associated with antipsychotics

\begin{tabular}{|c|c|c|c|c|}
\hline \multirow{2}{*}{ Variable } & \multicolumn{2}{|c|}{ Model 1} & \multicolumn{2}{|c|}{ Model 2} \\
\hline & Adjusted OR $(95 \% \mathrm{Cl})$ & $p$ value & Adjusted OR (95\% Cl) & $p$ value \\
\hline Age $(y r)$ & $1.00(1.00-1.00)$ & 0.318 & $1.00(1.00-1.00)$ & 0.956 \\
\hline \multicolumn{5}{|l|}{ Sex } \\
\hline Female & 1.00 & & 1.00 & \\
\hline Male & $1.00(0.91-1.10)$ & 0.951 & $1.04(0.93-1.17)$ & 0.452 \\
\hline \multicolumn{5}{|l|}{$\mathrm{CCl}$} \\
\hline 0 & 1.00 & & 1.00 & \\
\hline $1-2$ & $1.50(1.35-1.67)$ & $<0.001$ & $1.49(1.31-1.69)$ & $<0.001$ \\
\hline$\geq 3$ & $1.97(1.60-2.43)$ & $<0.001$ & $2.02(1.58-2.58)$ & $<0.001$ \\
\hline \multicolumn{5}{|c|}{ Use of antipsychotics } \\
\hline \multicolumn{5}{|c|}{ Typical and/or atypical } \\
\hline Past use & 1.00 & & - & \\
\hline Continue use & $1.55(1.40-1.72)$ & $<0.001$ & - & \\
\hline \multicolumn{5}{|l|}{ Typical } \\
\hline Past use & - & & 1.00 & \\
\hline Continue use & - & & $1.70(1.51-1.91)$ & $<0.001$ \\
\hline \multicolumn{5}{|l|}{ Atypical } \\
\hline Past use & - & & 1.00 & \\
\hline Continue use & - & & $1.43(1.28-1.60)$ & $<0.001$ \\
\hline
\end{tabular}

$\mathrm{Cl}$, confidence interval; $\mathrm{CCl}$, Charlson comorbidity index.

Model 1 was analyzed for using of typical and atypical antipsychotics. Model 2 was analyzed for using of typical and atypical antipsychotics, respectively.

years in the refracture group, and $41.4 \pm 14.6$ years in the control refracture group. Patients in the refracture group had greater physical comorbidities $(p<0.001)$. Continuous use of antipsychotics was found in $79.3 \%$ of the refracture patients, and $69.4 \%$ in controls $(p<0.001)$. Stratified analyses showed $49.9 \%$ of refracture patients conti- 
nuously used typical antipsychotics, and $61.1 \%$ atypical antipsychotics; for controls, the corresponding numbers were $37.2 \%$ and $54 \%(p<0.001$ and 0.047 , respec- tively) (Table 3).

Table 4 summarizes the relationship between refracture development and antipsychotic types used for these

Table 3. Characteristics of schizophrenia patients with and without refractures

\begin{tabular}{|c|c|c|c|c|c|c|}
\hline Variable & $\begin{array}{c}\text { Total } \\
(\mathrm{n}=915)\end{array}$ & $\begin{array}{l}\text { Without refracture } \\
\qquad(\mathrm{n}=422)\end{array}$ & $\begin{array}{l}\text { With refracture } \\
\qquad(n=493)\end{array}$ & $p$ value & $\begin{array}{l}\text { Crude OR } \\
(95 \% \mathrm{Cl})\end{array}$ & $p$ value \\
\hline Age (yr) & $44.1 \pm 15.7$ & $43.2 \pm 15.4$ & $44.9 \pm 16.0$ & $0.122 *$ & $1.01(1.00-1.01)$ & 0.122 \\
\hline Sex & & & & 0.574 & & \\
\hline Female & $445(48.6)$ & $201(47.6)$ & $244(49.5)$ & & 1.00 & \\
\hline Male & $470(51.4)$ & $221(52.4)$ & $249(50.5)$ & & $0.93(0.72-1.20)$ & 0.574 \\
\hline $\mathrm{CCl}$ & & & & $<0.001$ & & \\
\hline 0 & $529(57.8)$ & $272(64.5)$ & $257(52.1)$ & & 1.00 & \\
\hline $1-2$ & 295 (32.2) & $122(28.9)$ & $173(35.1)$ & & $1.50(1.13-2.00)$ & 0.006 \\
\hline$\geq 3$ & $91(9.9)$ & $28(6.6)$ & $63(12.8)$ & & $2.38(1.48-3.83)$ & $<0.001$ \\
\hline \multicolumn{7}{|l|}{ Use of antipsychotics } \\
\hline Typical and/or atypical & & & & 0.001 & & \\
\hline Past use & $231(25.2)$ & $129(30.6)$ & $102(20.7)$ & & 1.00 & \\
\hline Continue use & $684(74.8)$ & $293(69.4)$ & $391(79.3)$ & & $1.69(1.25-2.28)$ & 0.001 \\
\hline Typical & & & & $<0.001$ & & \\
\hline Past use & 505 (55.9) & $260(62.8)$ & $245(50.1)$ & & 1.00 & \\
\hline Continue use & $398(44.1)$ & $154(37.2)$ & $244(49.9)$ & & $1.68(1.29-2.20)$ & $<0.001$ \\
\hline Atypical & & & & 0.047 & & \\
\hline Past use & $319(42.3)$ & $163(46.0)$ & $156(38.9)$ & & 1.00 & \\
\hline Continue use & $436(57.7)$ & $191(54.0)$ & $245(61.1)$ & & $1.34(1.00-1.79)$ & 0.048 \\
\hline
\end{tabular}

Values are presented as mean \pm standard deviation or number $(\%)$.

$\mathrm{OR}$, odds ratio; $\mathrm{Cl}$, confidence interval; $\mathrm{CCl}$, Charlson comorbidity index.

${ }^{*} t$ test; chi-squared test or Fisher's exact test for all other $p$ value.

Table 4. Adjusted odds ratio (OR) of refractures associated with antipsychotics

\begin{tabular}{|c|c|c|c|c|}
\hline \multirow{2}{*}{ Variable } & \multicolumn{2}{|c|}{ Model 1} & \multicolumn{2}{|c|}{ Model 2} \\
\hline & Adjusted OR (95\% Cl) & $p$ value & Adjusted OR $(95 \% \mathrm{Cl})$ & $p$ value \\
\hline Age (yr) & $1.00(0.99-1.01)$ & 0.480 & $1.00(0.99-1.01)$ & 0.740 \\
\hline \multicolumn{5}{|l|}{ Sex } \\
\hline Female & 1.00 & & 1.00 & \\
\hline Male & $1.06(0.79-1.44)$ & 0.687 & $1.00(0.71-1.40)$ & 0.978 \\
\hline \multicolumn{5}{|l|}{$\mathrm{CCl}$} \\
\hline 0 & 1.00 & & 1.00 & \\
\hline $1-2$ & $1.45(1.08-1.95)$ & 0.015 & $1.43(1.03-1.99)$ & 0.035 \\
\hline$\geq 3$ & $2.31(1.41-3.77)$ & 0.001 & $2.97(1.68-5.25)$ & $<0.001$ \\
\hline \multicolumn{5}{|l|}{ Use of antipsychotics } \\
\hline \multicolumn{5}{|c|}{ Typical and/or atypical } \\
\hline Past use & 1.00 & & - & \\
\hline Continue use & $1.70(1.25-2.31)$ & 0.001 & - & \\
\hline \multicolumn{5}{|l|}{ Typical } \\
\hline Past use & - & & 1.00 & \\
\hline Continue use & - & & $1.84(1.35-2.50)$ & $<0.001$ \\
\hline \multicolumn{5}{|l|}{ Atypical } \\
\hline Past use & - & & 1.00 & \\
\hline Continue use & - & & $1.44(1.06-1.95)$ & 0.019 \\
\hline
\end{tabular}

$\mathrm{Cl}$, confidence interval; $\mathrm{CCl}$, Charlson comorbidity index.

Model 1 was analyzed for using of typical and atypical antipsychotics. Model 2 was analyzed for using of typical and atypical antipsychotics, respectively. 
patients. Risks of refracture were higher in continuous users $(\mathrm{OR}=1.70 ; 95 \% \mathrm{Cl}, 1.25-2.31)$ compared with past users (after adjustments). Similarly, increased odds of refracture were found in continuous use of antipsychotics, regardless of the drug types: typical $(\mathrm{OR}=1.84 ; 95 \% \mathrm{Cl}$, $1.35-2.50)$, and atypical $(\mathrm{OR}=1.44 ; 95 \% \mathrm{Cl}, 1.06-$ 1.95) (Table 4).

\section{Use of Individual Antipsychotics and Risks of Refracture}

The use of different kinds of antipsychotic and the risk of refracture were further analyzed. Compared to past users, higher odds of developing refractures were found among continuous users in the typical group of chlorpromazine $(\mathrm{OR}=2.45 ; 95 \% \mathrm{Cl}, 1.14-5.25)$, and in the atypical group of risperidone $(\mathrm{OR}=1.48 ; 95 \% \mathrm{Cl}, 1.01-$ 2.16) and zotepine $(\mathrm{OR}=2.15 ; 95 \% \mathrm{Cl}, 1.06-4.36)$ (Supplementary Table 1; available online).

\section{DISCUSSION}

We presented here the first population-based study assessing the association between antipsychotic medications and risks of fracture and refracture in the Asian schizophrenic population. We found that their prolonged use of antipsychotics, regardless of types, was positively associated with increased risks of fractures.

Our findings are consistent with previous reports that schizophrenic patients treated with antipsychotics have higher risks of bone fractures $[8,9,13,14]$. Earlier studies have reported that patients receiving typical antipsychotics had greater risks of hip fractures [13]. Other studies also found higher risks of falls with atypical antipsychotics [15], as well as more nonvertebral osteoporotic and hip fractures $[15,16]$. It is likely that underlying cause of fractures is falls.

Antipsychotics are dopamine $\mathrm{D}_{2}$ receptor antagonists, and may block prolactin secretion. It can inhibit hypothalamopituitary-gonadal axis, and consequently causing hyperprolactinemia and low BMD. Elevated serum prolactin can suppress secretion of gonadotropin-releasing hormone $(\mathrm{GnRH})$ form hypothalamus. In turn, low GnRH results in a reduced secretion of luteinizing hormone and follicle-stimulating hormone from pituitary gland, reducing levels of estradiol, progesterone and testosterone, and leading abnormal bone metabolism and osteoporosis $[4,7,17]$. Schizophrenic patients under prolonged anti- psychotic treatments have lower BMD and more pathological fractures [18]. Several epidemiologic studies showed a dose-response association of antipsychotics consumption with fracture risks in a Danish population [11], and with hip fracture risks among nursing home residents [18]. Thus, higher risks of fracture development of schizophrenic patients under treatments are one consequence of long-term antipsychotic medications.

Those patients with co-existing comorbid conditions and severe diseases may have even greater chances for fracture development. A recent study has reported higher risks of fracture in schizophrenic patients with poor physical functions and in hospitals [19]. Advanced ages may also be associated with falls due to lower BMD and age-driven decline in physical functions, leading to more fractures [19]. Rigler et al. [18], reported that for elderly in nursing homes, initiating antipsychotic treatment is linked to higher fracture risks, but the risks are similar across common antipsychotic uses. Liperoti et al. [19], reported that elderly patients receiving either typical or atypical antipsychotics have greater risks of hospitalization for femur fractures. Disease severity and old ages are likely related to more fractures in these schizophrenic patients.

In the present study, patients receiving continuous use of antipsychotics had more refractures. Bone tissue is continually being formed and resorbed, which called remodeling. It controlled by many factors, decline of estrogen levels leads to the acceleration of BMD depends loss, which resulted from loss of estrogen's bone protective effect. Thus, stable BMD depends on the balance of bone resorption and formation [18]. An earlier metaanalysis study reported that patients with longer duration of antipsychotic use, their bone healing is poorer, and that likely increases the risk of refractures [8]. Previous studies on the relations of neurotransmitter receptor binding affinity suggested a possible role of osteoporosis that underlies the association between antipsychotics and fracture $[4,7,13]$. The underlying mechanism could be due to continue effects of antipsychotic treatments, which by elevating prolactine levels towards osteoporosis, lower bone miner density and poor bone healing $[17,18]$, and then predisposing falls and refractures [15]. In addition to the antipsychotic medication, refracture probability is higher in patients with previous fractures and severe osteoporosis [20]. In brief, these factors partially explain the association between continuous use of antipsychotics and the 
risk of refracture in patients with prior fractures.

Despite several reports on relating antipsychotic uses with falls and fractures, limited knowledge was available relating typical and atypical antipsychotics with refractures. Our subgroup analyses showed that the continuous use of three drugs (chlorpromazine, risperdal, and zotepine) was related to highest risks of refracture. Differences in results across drugs could be due to differences in dosages and duration of pharmacological agents [14].

The strength of this study is the large number of schizophrenic patients we analyzed, and the small patient selection bias due to the nested case-control study design. In addition, this study provides the evidence that use of typical and atypical antipsychotics may be associated with increased risk of refracture development in schizophrenia patients.

Several limitations of our study should be noted. First, information regarding the sites of fracture and refracture were not available in our database. Second, detailed clinical information and laboratory as bone mineral score and lifestyle data were also not available and therefore these factors were not controlled. Several potential confounders such as obesity, family history of osteoporosis, accident fall down, poor function, ignore personal issues cause of difficult diagnosis of disease and substance uses (including smoking and alcohol consumptions), were also not available. Third, the use of different classes of antipsychotics among schizophrenia patients may increase their risk of fracture and refracture. Moreover, prescription of benzodiazepines often suggested in schizophrenia for insomnia and agitation. It may also related to fall and fracture, however, we were not able to demonstrate any association between polypharmacy status and fracture and refracture developments. Fourth, the generalizability of these results to other racial and ethnic groups is pending on further studies.

We showed that the continuous use of antipsychotic was associated with higher risks of fracture and refracture among schizophrenic patients receiving either typical or atypical antipsychotics. In addition, highest risks of developing refractures were observed among patients with continuous use of three drugs: chlorpromazine, risperdal and zotepine. Our findings suggested that clinicians should take into consideration the risk of developing fracture and refracture for patients receiving continuous antipsychotics. In prescription choices, clinicians may avoid chlorpromazine, risperdal and zotepine in fracture patients to prevent secondary fractures if they have more comorbidities and bone mineral density should be carefully monitored when this kind of medication is prescribed.

\section{Conflicts of Interest}

No potential conflict of interest relevant to this article was reported.

\section{Author Contributions}

Conceptualization: Ching-Min Kuo, Wei-Jen Liao and Ping-Wing Lui. Data acquisition: Ching-Heng Lin. Formal analysis: Chun-Che Huang and Ching-Heng Lin. Writingoriginal draft: Ching-Min Kuo and Wei-Jen Liao. Writingreview \& editing: Chun-Che Huang, Tsuo-Hung Lan, Ching-Heng Lin, Shun-Ping Wang, Cheng-Hung Lee and Ping-Wing Lui. Supervision: Ping-Wing Lui.

\section{ORCID}

Ching-Min Kuo https://orcid.org/0000-0002-5839-8440

Wei-Jen Liao

Chun-Che Huang https://orcid.org/0000-0003-0339-3615

Tsuo-Hung Lan https://orcid.org/0000-0002-2796-1026

Ching-Heng Lin https://orcid.org/0000-0002-2450-6108

Shun-Ping Wang https://orcid.org/0000-0002-7688-1671

Cheng-Hung Lee https://orcid.org/0000-0002-4176-2461

Ping-Wing Lui https://orcid.org/0000-0002-2918-2413

\section{REFERENCES}

1. McGrath J, Saha S, Chant D, Welham J. Schizophrenia: a concise overview of incidence, prevalence, and mortality. Epidemiol Rev 2008;30:67-76.

2. Kahn RS, Sommer IE, Murray RM, Meyer-Lindenberg A, Weinberger DR, Cannon TD, et al. Schizophrenia. Nat Rev Dis Primers 2015;1:15067.

3. Yang YH, Yeh EK, Hwu HG. Prevalences of schizophrenia, bipolar disorder, and depressive disorders in community between Taiwan and other countries. Taiwan J Psychiatry 2018; 26:77-87.

4. Kishimoto T, De Hert M, Carlson HE, Manu P, Correll CU. Osteoporosis and fracture risk in people with schizophrenia. Curr Opin Psychiatry 2012;25:415-429.

5. Fleischhacker WW, Meise U, Günther V, Kurz M. Compliance with antipsychotic drug treatment: influence of side effects. Acta Psychiatr Scand Suppl 1994;382:11-15.

6. De Hert M, Detraux J, Stubbs B. Relationship between antipsychotic medication, serum prolactin levels and osteopo- 
rosis/osteoporotic fractures in patients with schizophrenia: a critical literature review. Expert Opin Drug Saf 2016;15:809823.

7. Maguire GA. Prolactin elevation with antipsychotic medications: mechanisms of action and clinical consequences. J Clin Psychiatry 2002;63 Supp/ 4:56-62.

8. Papola D, Ostuzzi G, Thabane L, Guyatt G, Barbui C. Antipsychotic drug exposure and risk of fracture: a systematic review and meta-analysis of observational studies. Int Clin Psychopharmacol 2018;33:181-196.

9. Bakken MS, Schjøtt J, Engeland A, Engesaeter LB, Ruths S. Antipsychotic drugs and risk of hip fracture in people aged 60 and older in Norway. J Am Geriatr Soc 2016;64:1203-1209.

10. Koponen M, Taipale H, Lavikainen P, Tanskanen A, Tiihonen J, Tolppanen AM, et al. Antipsychotic use and the risk of hip fracture among community-dwelling persons with Alzheimer's disease. J Clin Psychiatry 2017;78:e257-e263.

11. Chu CS, Chou PH, Chen YH, Huang MW, Hsu MY, Lan TH, et al. Association between antipsychotic drug use and cataracts in patients with bipolar disorder: a population-based, nested case-control study. J Affect Disord 2017;209:86-92.

12. Wu CS, Chang CM, Tsai YT, Huang YW, Tsai HJ. Antipsychotic treatment and the risk of hip fracture in subjects with schizophrenia: a 10-year population-based case-control study. J Clin Psychiatry 2015;76:1216-1223.

13. Lee SH, Hsu WT, Lai CC, Esmaily-Fard A, Tsai YW, Chiu CC, et al. Use of antipsychotics increases the risk of fracture: a systematic review and meta-analysis. Osteoporos Int 2017;28:
1167-1178

14. Fraser LA, Liu K, Naylor KL, Hwang YJ, Dixon SN, Shariff SZ, et al. Falls and fractures with atypical antipsychotic medication use: a population-based cohort study. JAMA Intern Med 2015;175:450-452.

15. Pouwels S, van Staa TP, Egberts AC, Leufkens HG, Cooper C, de Vries F. Antipsychotic use and the risk of hip/femur fracture: a population-based case-control study. Osteoporos Int 2009;20:1499-1506.

16. Rady A, Elsheshai A, Elkholy O, Abouelwafa H, Eltawil M. Long term use of antipsychotics and adverse effects on bone density. Neuropsychiatry (London) 2018;8:1559-1563.

17. Uchida S, Ichinose T, lizuka Y, Okamura K, Shitara H, Yamazaki M, et al. Osteopenia and the physical function in Japanese patients with schizophrenia. Arch Osteoporos 2017;12:93.

18. Rigler SK, Shireman TI, Cook-Wiens GJ, Ellerbeck EF, Whittle JC, Mehr DR, et al. Fracture risk in nursing home residents initiating antipsychotic medications. J Am Geriatr Soc 2013;61: 715-722.

19. Liperoti R, Onder G, Lapane KL, Mor V, Friedman JH, Bernabei $\mathrm{R}$, et al. Conventional or atypical antipsychotics and the risk of femur fracture among elderly patients: results of a casecontrol study. J Clin Psychiatry 2007;68:929-934.

20. Chao CT, Yang RS, Huang WJ, Tsai KS, Chan DD. Risk factors for poor functional recovery, mortality, recurrent fractures, and falls among patients participating in a fracture liaison service program. J Am Med Dir Assoc 2019;20:1129-1136.e1. 